\title{
p-HARMONIC MORPHISMS, COHOMOLOGY CLASSES AND SUBMERSIONS
}

\author{
BANG-YEN CHEN AND SHIHSHU WALTER WEI*
}

\begin{abstract}
By studying cohomology classes that are related with $p$-harmonic morphisms, we extend some previous results of the first author on Riemannian submersions with minimal fibers to $n$-harmonic morphisms which are submersions.
\end{abstract}

\section{Introduction}

In the study of topology on a compact Riemannian manifold $M$, it is well-known that nontrivial fundamental groups $\pi_{1}(M)$, homology classes, and cohomology classes can be represented by stable closed geodesics, stable minimal currents, and harmonic forms on $M$, by Cartan's Theorem ([3]), Federer-Fleming's Theorem ([7]), and Hodge Theorem $([8])$, respectively. In an analogous spirit, it is shown in [13] that homotopy classes can be represented by $p$-harmonic maps (For definition and examples of $p$-harmonic maps, see e.g. [14]):

Theorem A. If $N$ is a compact Riemannian manifold, then for any positive integer $i$, each class in $\pi_{i}(N)$ can be represented by a $C^{1, \alpha}$ p-harmonic map $u_{0}$ from $S^{i}$ into $N$ minimizing $p$-energy in its homotopy class for any $p>i$.

Further applications and homotopically vanishing theorems are given in $[13,16]$. As a $p$-harmonic morphism by definition, carries germs of $p$-harmonic functions to germs of $p$-harmonic functions, and can be characterized as a horizontally weak conformal $p$ harmonic map (cf. Theorem 4.), a $p$-harmonic morphism should be also linked with topology in a certain way.

On the other hand, B.-Y. Chen provides the following link between Riemannian submersions and minimal immersions with cohomology class (cf. [5]):

Theorem B. Let $\pi: M \rightarrow B$ be a Riemannian submersion with minimal fibers and orientable base manifold $B$. If $M$ is a closed manifold with cohomology class $H^{b}(M, \mathbf{R})=$

Corresponding author: Shihshu Walter Wei.

2000 Mathematics Subject Classification. Primary 58E20; Secondary 53C42

Key words and phrases. Cohomology class, p-harmonic morphism, minimal submanifold, submersion, Riemannian submersion.

* Research was partially supported by NSF Award No DMS-0508661. 
$0, b=\operatorname{dim} B$, then the horizontal distribution $\mathcal{H}$ of the Riemannian submersion is never integrable. Thus the submersion $\pi$ is always non-trivial.

Let $M$ and $B$ be differentiable manifolds and let $\phi: M \rightarrow B$ be a differentiable map between them. The map $\phi$ is called a submersion if, at each point $x \in M$, the differential $d \phi_{x}$ is a surjective linear map. A closed manifold is a compact manifold without boundary.

Let $\pi: M \rightarrow B$ be a submersion between two Riemannian manifolds. For each $x \in B$, $\pi^{-1}(x)$ is an $(n-b)$-dimensional submanifold of $M$, which is called a fiber. A vector field on $M$ is called vertical if it is always tangent to fibers; and it is called horizontal if it is always orthogonal to fibers in $B$.

The simplest type of Riemannian submersions is the projection of a Riemannian product manifold on one of its factors. For such Riemannian submersions, both horizontal and vertical distributions are totally geodesic distributions, i.e., both distributions are completely integrable and their leaves (i.e., integrable submanifolds) are totally geodesic submanifolds.

A submersion $\pi: M \rightarrow B$ between two Riemannian manifolds is said to be nontrivial if its horizontal and vertical distributions are not both totally geodesic distribution. And a submersion $\pi: M \rightarrow B$ between two Riemannian manifolds is called a Riemannian submersion if the differential $d \pi$ preserves the length of horizontal vector fields.

The purpose of this article is to connect and extend the two seemingly unrelated areas of $p$-harmonic morphisms and cohomology classes. More precisely, we prove the following.

Theorem 1. Let $u: M \rightarrow N$ be an $n$-harmonic morphism which is a submersion. If $M$ is a closed manifold with cohomology class $H^{n}(M, \mathbf{R})=0$ with $n=\operatorname{dim} N$, then the horizontal distribution $\mathcal{H}$ of $u$ is never integrable. Thus the submersion $u$ is always non-trivial.

Theorem 1. recaptures Theorem B, when $u: M \rightarrow B$ is a Riemannian submersion with minimal fibers and orientable base manifold $B$. To see this, we note that in general, $n$-harmonic morphisms into $n$-manifolds which are submersions but not necessarily Riemannian submersions with minimal fibers(cf. Section 5). On the other hand, a Riemannian submersion into an $n$-manifold with minimal fibers is an $n$-harmonic morphism which is a submersion by a result of [2] (cf. Theorem 4.).

Theorem 2. Let $u: M \rightarrow N$ be an $n$-harmonic morphism which is a submersion from a closed manifold $M$ with $n=\operatorname{dim} N$. Then the pull back of the volume element of $N$ is a harmonic $n$-form if and only if the horizontal distribution $\mathcal{H}$ of $u$ is completely integrable.

This Theorem recaptures a result of [5] (cf. Theorem 3.).

\section{Minimal submanifolds, submersions and $p$-harmonic morphisms}

We recall some related basic facts, notations, definitions, and formulas (see $[4,5,6]$ for details). 


\subsection{Basic formulas and equations}

Let $\tilde{M}$ be a Riemannian manifold with Levi-Civita connection $\tilde{\nabla}$. The tangent bundle of $\tilde{M}$ is denoted by $T \tilde{M}$, and the (infinite dimensional) vector space of smooth sections of a smooth vector bundle $E$ is denoted by $\Gamma(E)$.

Let $M$ be a submanifold of dimension $n \geq 2$ in $\tilde{M}$. Denote by $\nabla$ and $D$, the LeviCivita connection and the normal connection of $M$, respectively. For each normal vector $\xi \in T_{p}^{\perp} M, p \in M$, the shape operator $A_{\xi}$ is a symmetric endomorphism of the tangent space $T_{p} M$ at $p$. Then the shape operator and the second fundamental form $h$ are related by

$$
\langle h(X, Y), \xi\rangle=\left\langle A_{\xi} X, Y\right\rangle
$$

for $X, Y$ tangent to $M$ and $\xi$ normal to $M$.

The formulas of Gauss and Weingarten are given respectively by (cf. [4])

$$
\begin{aligned}
& \tilde{\nabla}_{X} Y=\nabla_{X} Y+h(X, Y), \\
& \tilde{\nabla}_{X} \xi=-A_{\xi} X+D_{X} \xi
\end{aligned}
$$

for tangent vector fields $X, Y$ and normal vector field $\xi$ on $M$.

\subsection{Definitions}

The mean curvature vector field of a submanifold $M$ in a Riemannian manifold is defined by

$$
H=\left(\frac{1}{n}\right) \text { trace } h
$$

A submanifold $M$ in $\tilde{M}$ is called totally geodesic (respectively, minimal) if the second fundamental form $h$ (respectively, the mean curvature vector field $H$ ) of $M$ vanishes identically.

\subsection{Riemannian submersions}

For Riemannian submersions we have the following result from [5].

Theorem 3. Let $\pi: M \rightarrow B$ be a Riemannian submersion from a closed manifold $M$ onto an orientable base manifold $B$. Then the pull back of the volume element of $B$ is harmonic if and only if the horizontal distribution $\mathcal{H}$ is integrable and fibers are minimal.

\section{3. p-harmonic morphisms}

A $C^{2}$ map $u: M \rightarrow N$ between two Riemannian manifolds is said to be a $p$-harmonic morphism if, for any p-harmonic function $f$ defined on an open set $V$ of $N$, the composition $f \circ u$ is $p$-harmonic on $u^{-1}(V)$. 
A $C^{2}$ map $u: M \rightarrow N$ between two Riemannian manifolds is called horizontally weak conformal if, for any $x$ such that $d u(x) \neq 0$, the restriction of $d u(x)$ to the orthogonal complement $H$ of $\operatorname{Ker}(d u(x))$ is conformal and surjective.

Recently, E. Loubeau and Burel-Loubeau obtain a characterization of a $p$-harmonic morphism:

Theorem 4. $[1,9] A C^{2}$ map $u: M \rightarrow N$ is a p-harmonic morphism with $p \in(1, \infty)$ if and only if it is a p-harmonic and horizontally weak conformal map.

In [2], P. Baird and S. Gudmundsson link $n$-harmonic morphism with minimal fibers:

Theorem 5. [2] Let $u: M \rightarrow N$ be a horizontally conformal submersion. Then $u$ is $n$-harmonic with $n=\operatorname{dim} N$ if and only if the fibers of $u$ are minimal in $M$.

\section{Proof of Theorems 1. and 2.}

By virtue of Theorems 4. and 5., an $n$-harmonic morphism $u$ which is a submersion with $n=\operatorname{dim} N$ has minimal fibers. Thus, we may proceed as in the proof of Theorem 3. (see [5] for details): Since the fibers are minimal submanifold of $N$ (hence (6.6) in [5] holds), the pull back of the volume element $\omega$ of $N$ is a co-closed $n$-form if and only if the horizontal distribution $\mathcal{H}$ of the submersion $u$ is integrable (hence (6.7) in [5] also holds). Furthermore, the pull back of the volume element $\omega$ of $N$ is also a closed form, since the exterior differentiation $d$ and the pull back $u^{*}$ commutes. Therefore, it follows that $\omega$ is a harmonic $n$-form if and only if the horizontal distribution $\mathcal{H}$ of $u$ is completely integrable. In case, $\omega$ is harmonic, it represents a nontrivial cohomology class $H^{n}(M, \mathbf{R})$ by Hodge Theorem. This proves Theorem 2 ..

Since each nonzero harmonic form represents a non-trivial cohomology class by Hodge Theorem, and since on a closed manifold a differential form is harmonic if and only if it is closed and co-closed, Theorem 1 . follows from Theorem 2 ..

\section{Examples of $p$-harmonic morphisms which are submersion, but not Riemannian submersions}

In this section, we provide some simple examples of $p$-harmonic morpisms which are submersion, but not Riemannian submersions.

Example 5.1. Let $\pi: \mathbb{E}^{k} \times \mathbb{E}^{n} \rightarrow \mathbb{E}^{n}$ be an orthogonal projection and let $\sigma_{n}: \mathbb{E}^{n} \rightarrow \mathbb{E}^{n}$ be a non-isometric conformal diffeomorphism, where $\mathbb{E}^{n}$ is the Euclidean $n$-space. Then $\sigma_{n} \circ \pi$ is an $n$-harmonic morphism which is a submersion with minimal fibers but is not a Riemannian submersion (cf. Example 4.9 in [11]).

Analogously, we have on compact manifolds 
Example 5.2. Let $\pi_{2}: S^{k} \times S^{n} \rightarrow S^{n}$ be an orthogonal projection and let $\gamma_{n}: S^{n} \rightarrow S^{n}$ be a non-isometric conformal diffeomorphism. Then $\gamma_{n} \circ \pi_{2}$ is an $n$-harmonic morphism which is a submersion with minimal fibers but is not a Riemannian submersion.

These examples are based on the fact that the composition of $p$-harmonic morphisms is a $p$-harmonic morphism, and a conformal map between equal dimensional $n$-manifolds, such as stereographic projections $u: \mathbb{E}^{n} \rightarrow S^{n}$ is an $n$-harmonic morphism (cf. [15, 11]).

\section{References}

[1] J. M. Burel and E. Loubeau, $p$-harmonic morphisms: the $1<p<2$ case and a non-trivial example, Contemp. Math. 308 (2002), 21-37.

[2] P. Baird and S Gudmundsson, p-harmonic maps and minimal submanifolds, Math. Ann. 294 (1992), 611-624.

[3] E. Cartan, Lecons sur la Geométrie des Espaces de Riemann, 2nd edition, Gauthier-Villars, Paris, 1951.

[4] B.-Y. Chen, Geometry of Submanifolds, M. Dekker, New York, 1973.

[5] B.-Y. Chen, Riemannian submersions, minimal immersions and cohomology class Proc. Japan Acad. Ser. A Math. Sci. 81 (2005), 162-167.

[6] B.-Y. Chen and S. W. Wei, Submanifolds of warped product manifolds $I \times_{f} S^{m-1}(k)$ from a p-harmonic viewpoint, Bull. Transilv. Univ. Braşov Ser. III, 1(50) (2008), 59-78.

[7] H. Federer and W. Fleming, Normal and integral currents, Ann. of Math., 72 (1960) 458520

[8] W.V.D. Hodge, The theory and application of harmonic intergrals, Cambridge University Press, New York (1941) (2nd ed., 1952)

[9] E. Loubeau, On p-harmonic morphisms, Diff. Geom. Appl. 12 (2000), 219-229.

[10] B. O'Neill, Semi-Riemannian Geometry with Applications to Relativity, Academic Press, New York, 1982.

[11] Y. L. Ou and S. W. Wei, A classification and some constructions of p-harmonic morphisms, Beiträge Algebra Geom. 45 (2004) 637-647.

[12] A. V. Pogorelov, On the stability of minimal surfaces, Soviet Math. Dokl. 24 (1981), 274276 .

[13] S. W. Wei, Representing Homotopy Groups and Spaces of Maps by p-harmonic maps, Indiana Univ. Math. J. 47 (1998), 625-670.

[14] S. W. Wei, p-Harmonic geometry and related topics, Bull. Transilv. Univ. Brasov Ser. III 1(50) (2008), 415-453.

[15] S.W. Wei, J.F. Li, and L. Wu, Generalizations of the Uniformization Theorem and Bochner's Method in p-Harmonic Geometry, Proceedings of the 2006 Midwest Geometry Conference, Commun. Math. Anal. 2008, Conference 1, 46-68

[16] S. W. Wei, and L. Wu, Homotopy groups and p-Harmonic Maps, Commun. Math. Anal. 2008, Conference 1, 40-45.

Department of Mathematics, Michigan State University, East Lansing, Michigan 48824-1027, U.S.A. 
E-mail: bychen@math.msu.edu

Department of Mathematics, University of Oklahoma, Norman, Oklahoma 73019-0315, U.S.A.

E-mail: wwei@ou.edu 